\author{
Daniel E. Bailey, University of Colorado, Presider
}

\title{
Teaching experimental psychology with on-line computers
}

\author{
ARTHUR O. CROMER \\ University of Louisville, Louisville, Kentucky 40208
}

The purpose of this paper is to describe the use of on-line computers in the teaching of experimental methodology. Computer models are featured.

The use of on-line computers for experimental control has become an accepted part of psychological research technique, and computer-assisted instruction (CAI) has become an accepted, if not widely practiced, part of educational technique. Aside from CAI, computers have mainly been used in the psychology classroom as an elaborate calculator to assist in data reduction or as a mechanism to be studied in order to improve laboratory control. This paper describes a new use of computers, which is as a tool to teach methodology. not content, by the method of discovery learning, and this supplement and complement, not replace. the traditional methods.

An undergraduate experimental psychology course may have as its goals, "the teaching of experimental methods, practice in the application of the scientific methods to research problems, and practice in designing, conducting, and analyzing of experiments." These goals necessarily involve the conduct of a number of experiments which simultaneously teach the content area involved, the methods unique to that area. such as how to handle a rat without getting bitten, as well as how to plan and carry out experiments. One drawback to this approach is that some of the actual experiments take so long that the number of experiments of that type that can be conducted during a term is limited, usually to a single example. If the student wishes to rerun the experiment, to add needed controls. or because his animal died. etc.. the time constraints preclude this logical follow-up to the original experiment. What seems to be emphasized to the student is not how experiments should be run. but what a haphazard business research is.

At the University of Louisville, a revision to the traditional course was planned during the first part of 1971 and implemented in the fall of that year. The original plan was to have three of our five sections use the computer-assisted program while the other two. taught by older full professors. would use the traditional approach, thus providing a ready-made experiment. Unfortunately, by the middle of the first semester, all sections were using the computer, at least partially, and the opportunity for direct comparison was lost.

The course, as presently implemented, involves about four computer models interspersed with at least one live experiment and several live demonstrations. The models available are: a verbal dominance situation, originally conceived by Johnson (1971) at Earlham College; a survey-type study of the causes of schizophrenia, adopted from a model used at Michigan (Main \& Head, 1971); an imprinting experiment, also based on a model developed at Michigan (Main \& Head, 1971); a study of the psychology of eating in overweight rats and people (Thurmond \& Cromer, 1972); a model of the effects on language discrimination in the cocktail party phenomenon (Thurmond \& Cromer, 1972); and a model investigating the tip-of the-tongue phenomenon. A typical model would require the student, after reading a background scenario of from 2 to 20 pages, to input a number of parameters to the model. These parameters are of the type: discrete (sex, method of diagnosis), continuous (age). general parameter (number of trials), or hidden $(\mathrm{X})$, and have default values or, in some cases, are randomized if defaulted. The computer model calculates the results, and data are then presented as if the specified experiment had just been run. Along with this output. most programs give an "experimental cost" based on the parameter values chosen. The student then may ask for summary statistics and the standard error of this run as compared to the preceding run, to aid in analysis. The models have from 6 to 12 variables and are usually used in increasing order of difficulty and complexity.

The models used at Michigan were written in FORTRAN IV and run in batch mode on the 360/67. These were rewritten in FORTRAN IV and run as overlayed programs on the 8K PDP-9 of the Psychology Department at the University of Louisville during the fall of 1971. A single terminal. connected as a remote console terminal. was made available in the classroum during class time only. While each model was a completely separate and distinct program. uniformity was incorporated in the form of parallel or identical subroutines and links in each program performing 
equivalent functions. There was a main program plus 10 subprograms scattered into four links. The links served the purposes of: input, checking parameter limits, actual model, and statistics. The program called upon a "hidden" data file that contained the legal limits for each parameter, the effects values to be used by the model, and the costs to be used in computing the "cost" for a particular run.

Because of the single terminal and tying up the departmental computer during "prime time," the models were reprogrammed in BASIC and run on a small time-shared computer (HP2000A) starting in the spring of 1972. In the process of conversion, a major revision was made to the program organization. The BASIC version consists of one generalized main program, to which is appended a unique module containing one or two models. The same main program thus services all models. In addition, instead of a separate "hidden" data file for each program, there is a single data file used by all models. In order to generate this data file, a separate program was created which, through a series of questions, allows the author to insert data for a new model in a matter of minutes. In the BASIC programs, there is provision for six types of variables. In addition to the five present in FORTRAN, a dependent-type variable has been added.

The use of the "hidden" variables was an attempt to make the models more realistic. Initially, the student is only aware of certain parameters or variables he can control in an experiment and is challenged to discover what an extra or " $X$ " variable must he. After suitable effort, he is told that " $X$ " contains an additional set of independent variables and he is given the opportunity to control them also, but there is still an elusive "X." At both stages, the "X" may be defaulted, in which case its effect is randomized or it may be named, a code number for it given, the allowable limits given, and a desired level specified. The key to the effect is in the code, which at present must be obtained from the instructor or laboratory assistant after describing the proposed experiment. By selecting certain codes, the " $\mathrm{X}$ " variable can be caused to have no effect (randomized) or a positive or negative linear, normal, exponential, etc., effect. Thus, the model, with the help of a human, can accommodate any variable a student may come up with.

An example of the way this " $X$ " variable works can be seen in the fat rat model. Initially, the students are aware of the independent variables: $N$ (number of $S s$ ), type of animal (rat or man), size of S (normal or obese), hypothalamic activity (unknown or functionally quiescent), hunger level (comfortable, empty stomach, epinephrine-simulated low hunger, or full preloaded stomach), amount of work to get food (none or moderate), emotionality (four levels, from lethargic through very strong reaction to disturbing conditions), and distractibility (seven levels, from low to high). They are told to discover the relationships among these variables and challenged to discover what variable or variables constitute the " $\mathrm{X}$ " or unknown factors. For man, the amount eaten ranges from a mean of $180 \mathrm{~g}$ to a mean of $560 \mathrm{~g}$, with a median of $260 \mathrm{~g}$. Of this, the $X$ variable accounts for $80-400 \mathrm{~g}$. If a student comes up with a variable, e.g., illumination level of food, which is judged to have a mildly rising linear effect, a code is given to the student in which the middle digit is 9 . Then, whatever value the student chooses for "X," the effects will range from +80 to +200 , depending on the particular value within the legal limits the student chooses. If, however, the student comes up with a variable judged to have no effect, a code is given to the student which has a middle digit of 1,3 , or 7 . Then, no matter what value of that variable the student chooses, the effect will be randomly chosen from the range of +80 to $+400 \mathrm{~g}$.

One of the most difficult, and as yet unsolved problems, has been how to evaluate the student's performance in such a class. Because the student no longer turns in a few reports of obviously incomplete research findings, but typically reports 50 studies per semester, the traditional reliance on grading research reports for technical quality rather than content no longer works. The emphasis has been to encourage the students first to work alone, then to form research teams. The research teams allow division of the work and discussion of results that cap increase the productivity considerably. Because of the complexity of the models, there is no single question clearly indicated, but rather a number of reasonable questions can be put together worthy of investigation. One consideration which may help to determine which questions the team will tackle is the "cost." Frequently, it is shown that the same, or more powerful, finding can be obtained at a reduced cost by careful planning of research strategy. The logical extension of two groups to many groups to factorial experiments is discovered by the students in an attempt to get "more for their money." There are even instances of students asking to be taught statistics to analyze their data, or reading up on the techniques on their own. This allows one evaluation of the student's performance to be in terms of "cost" to answer particular questions. Another evaluation technique has been the presentation of one or more relatively simple models to be "solved" in an examination. Here, the premium is on speed and quality of technique. The first is objective, the second rather subjective.

The most immediate change proposed to the present system is to allow permanent records of who used a model, what was done, total "cost," etc. This cannot be implemented on our present computer system without resorting to "tricks," and this would tend to reduce the exportability of the programs. In spite of this drawback, this change will probably be implemented before the spring of 1973.

Several ideas have been proposed for the further utilization of the simulation approach. Ex-students in this course have asked if there isn't anything else of the 
same type available to them. One idea would be to have a second-level course for undergraduates who would be given a certain amount of "funds" and formed up into "granting agencies" to review requests for funds for proposed research projects by the first-level classes. Other ideas involve using lots of additional models not for methodology, but to learn content in a variety of courses. An undergraduate student in Abnormal Psychology who relates to a "schizophrenic" simulation and a "manic" simulation wouldn't soon forget the differences. Or, the student who can actuaily try out various theoretical models of learning could internalize these models in far different ways than from only reading about them.

Graduate students could be required or optionally allowed to produce a model or models as a part of their regular courses instead of producing a research paper or term project. After putting together a model of a theory, the holes, strengths, weaknesses, and ambiguities of the theory could be abundantly clear. This might logically lead to the use of models of theories as a research technique that the students could use later.

At the University of Louisville, we plan to incorporate the present semester experimental psychology course and the present semester statistics course into a 1-year integrated course having a blend of both during the entire year. We are presently at work on computer demonstrations designed to make the concepts of statistics easier to grasp and to plan the curriculum for this combined course.

\section{REFERENCES}

Johnson, R. DATACALL: A computer-based simulation game for teaching strategy in scientific research. Proceedings of the Conference on Computers in the Undergraduate Curricula, Dartmouth, June 1971 .

Main, D. B.. \& Head, S. Computer simulations in the elementary psychology laboratory. Proceedings of the Conference on Computers in the Undergraduate Curricula, Dartmouth, June 1971.

Thurmond, J. B., \& Cromer, A. O. Towand the optimal use of computer simulations in teaching scientific research strategy. Proceedings of the Conference on Computers in the Undergraduate Curricula, Atlanta. June 1972.

\section{The effectiveness of direct graphical entry of topological and geometric data}

\author{
MELVIN R. CORLEY and JOHN J. ALLAN III \\ Department of Mechanical Engineering \\ Lniversity of Texas at Austin. Austin. Texas 78712
}

A study is described that was carried out to determine the feasibility of making interactive graphics terminals available for computer-aided instruction (CAI) in engineering and science courses.

One of the most necessary skills required of engineers has long been the ability to communicate graphically. Many problems in the engineering profession are presented in a graphical format, and solutions of such problems are also usually depicted graphically. Another skill required more recently is the ability to utilize the digital computer effectively to aid in the computational aspects of engineering analysis. This has presented some problems to engineering, since those problems which are best represented graphically must be encoded into some form of alphanumeric information which is compatible with the input capabilities of the computer used. In most batch processing systems. this usually means punching the data on cards. The use of digital computers has taken on more importance recently because of three developments: (1) MSI-LSI technology: (2) high-level programming language: and (3) multiprogramming and time-sharing operating systems. All of these are especially evident in the rapidly growing area of interactive computer graphics. Recently, we have seen the introduction of interactive graphics terminals that are capable of operating in a time-sharing environment.

The goal of this study was to determine the feasibility of making interactive graphics terminals available for CAI in engineering and science courses. Use of such terminals might be attrative from not only a pedagogical standpoint. but also for the practical aspects, since it is likely that these devices will become more and more commonly used as standard analytical and design aids. More specifically, this study sought to compare, in terms of speed and accuracy, various methods that could be used to input graphical data to a computer from a time-sharing graphics terminal.

The problem selected as a vehicle for comparing data entry techniques was one that is often faced by mechanical engineers in industry, piping flexibility analysis. This area of applied stress analysis is widely used in the chemical and petroleum process industries and involves knowledge of stress-strain relationships, mechanical design, and materials. The objective of piping flexibility analysis is to position the anchors and restraints in a pipe system at locations that will allow the pipe system to safely absorb loads induced by restrained linear thermal expansion. Such problems are invariably presented as schematic diagrams. A significant problem arises when the problem is to be submitted to a computer program for solution. The problem is to convert efficiently the topological and geometric data of 